\title{
Report on the first round of the Mock LISA Data Challenges
}

\author{
K A Arnaud ${ }^{1}$, G Auger ${ }^{2}$, S Babak ${ }^{3}$, J G Baker ${ }^{1}$, M J Benacquista ${ }^{4}$, \\ E Bloomer ${ }^{5}$, D A Brown ${ }^{6,7}$, J B Camp ${ }^{8}$, J K Cannizzo ${ }^{8}$, N Christensen ${ }^{9}$, \\ J Clark $^{5}$, N J Cornish ${ }^{10}$, J Crowder ${ }^{10,11}$, C Cutler ${ }^{7,11}$, L S Finn ${ }^{12}$, \\ H Halloin ${ }^{2}$, K Hayama ${ }^{4}$, M Hendry ${ }^{5}$, O Jeannin ${ }^{2}$, A Królak ${ }^{13}$, \\ S L Larson ${ }^{14}$, I Mandel ${ }^{6}$, C Messenger ${ }^{5}$, R Meyer ${ }^{15}$, S Mohanty ${ }^{4}$, \\ R Nayak ${ }^{4}$, K Numata ${ }^{8}$, A Petiteau ${ }^{2}$, M Pitkin ${ }^{5}$, E Plagnol ${ }^{2}$, \\ E K Porter ${ }^{3,10}$, R Prix ${ }^{3}$, C Roever ${ }^{15}$, A Stroeer ${ }^{16,17}$, \\ R Thirumalainambi ${ }^{18}$, D E Thompson ${ }^{18}$, J Toher ${ }^{5}$, R Umstaetter ${ }^{15}$,

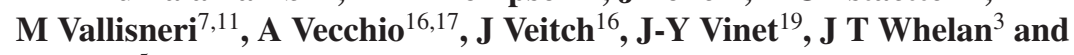 \\ G Woan ${ }^{5}$ \\ ${ }^{1}$ Gravitational Astrophysics Laboratory, NASA Goddard Space Flight Center, \\ 8800 Greenbelt Rd, Greenbelt, MD 20771, USA \\ 2 AstroParticule et Cosmologie (APC), UMR 7164, Université Paris 7 Denis Diderot, \\ 10 rue Alice Domon et Léonie Duquet, F-75205 Paris Cedex 13, France \\ ${ }^{3}$ Max-Planck-Institut für Gravitationsphysik (Albert-Einstein-Institut), Am Mühlenberg 1, \\ D-14476 Golm bei Potsdam, Germany \\ ${ }^{4}$ Center for Gravitational Wave Astronomy, University of Texas at Brownsville, Brownsville, \\ TX 78520, USA \\ ${ }^{5}$ Department of Physics and Astronomy, University of Glasgow, Glasgow G12 8QQ, UK \\ ${ }^{6}$ LIGO Laboratory, California Institute of Technology, Pasadena, CA 91125, USA \\ ${ }^{7}$ Theoretical Astrophysics, California Institute of Technology, Pasadena, CA 91125, USA \\ ${ }^{8}$ Laboratory for Gravitational Physics, Goddard Space Flight Center, Greenbelt, MD 20771, USA \\ ${ }_{9}^{9}$ Physics and Astronomy, Carleton College, Northfield, MN, USA \\ ${ }^{10}$ Department of Physics, Montana State University, Bozeman, MT 59717, USA \\ ${ }^{11}$ Jet Propulsion Laboratory, California Institute of Technology, Pasadena, CA 91109, USA \\ ${ }^{12}$ Center for Gravitational Wave Physics, The Pennsylvania State University, University Park, \\ PA 16802, USA \\ ${ }^{13}$ Institute of Mathematics, Polish Academy of Science, Warsaw, Poland \\ ${ }^{14}$ Department of Physics, Weber State University, 2508 University of Circle, Ogden, UT 84408, \\ USA \\ ${ }^{15}$ Department of Statistics, The University of Auckland, Auckland, New Zealand \\ ${ }^{16}$ School of Physics and Astronomy, University of Birmingham, Edgbaston, Birmingham \\ B15 2TT, UK \\ ${ }^{17}$ Department of Physics and Astronomy, Northwestern University, Evanston, IL 60208, USA \\ ${ }^{18}$ NASA Ames Research Center, Moffett Field, CA, USA \\ ${ }^{19}$ Department ARTEMIS, Observatoire de la Côte d'Azur, BP 429, 06304 Nice, France \\ E-mail: benacquista@phys.utb.edu
}

Received 30 March 2007, in final form 2 April 2007

Published 19 September 2007

Online at stacks.iop.org/CQG/24/S529 


\begin{abstract}
The Mock LISA Data Challenges (MLDCs) have the dual purpose of fostering the development of LISA data analysis tools and capabilities, and demonstrating the technical readiness already achieved by the gravitational-wave community in distilling a rich science payoff from the LISA data output. The first round of MLDCs has just been completed: nine challenges consisting of data sets containing simulated gravitational-wave signals produced either by galactic binaries or massive black hole binaries embedded in simulated LISA instrumental noise were released in June 2006 with deadline for submission of results at the beginning of December 2006. Ten groups have participated in this first round of challenges. All of the challenges had at least one entry which successfully characterized the signal to better than $95 \%$ when assessed via a correlation with phasing ambiguities accounted for. Here, we describe the challenges, summarize the results and provide a first critical assessment of the entries.
\end{abstract}

PACS numbers: 95.55.Ym, 04.80.Nn, 05.45.Tp, 07.05.Kf

\title{
1. Introduction
}

At the LISA International Science Team (LIST) meeting of December 2005, the Working Group on Data Analysis (LIST-WG1B) decided to organize several rounds of MLDCs with the dual purposes of (i) fostering the development of LISA data analysis tools and capabilities and (ii) determining the technical readiness already achieved by the gravitational-wave community for distilling a rich science payoff from the LISA data output. These challenges are meant to be blind tests, but not contests. The intent is to encourage the quantitative comparison of results, analysis methods and implementations.

A MLDC Task Force was constituted at the beginning of 2006 and has been working since then to formulate challenge problems, develop standard models of the LISA mission and gravitational-wave $(\mathrm{GW})$ sources, provide computing tools (e.g. LISA response simulators and source waveform generators), establish criteria for the evaluation of the analyses and provide any technical support necessary to the challenge participants. The first round of challenges involve the distribution of several data sets, encoded in a simple standard format, and containing combinations of realistic simulated LISA noise with the signals from one or more GW sources with parameters which were unknown to the participants. The participants were then asked to return the maximum amount of information about the sources and to produce technical notes detailing their work.

The release of the first round of challenge data sets was announced in June 2006 at the Sixth LISA International Symposium hosted by the Goddard Space Flight Center in Greenbelt, Maryland [1, 2]. John Baker (a member of the MLDC Task Force who did not participate in the first round) was appointed as MLDC1 coordinator. The coordinator was responsible for generating the challenge data sets, receiving the results from the participants, and posting both the key data files and results as soon as possible after the submission deadline of 4 December 2006.

The challenge data sets include a total of 9 years long data sets which are described in detail on the MLDC website [3], the Task Force wiki [4] and the Omnibus document for Challenge 1 [5]. The challenge data sets are broadly grouped into three categories: (1.1) white 
Table 1. Groups that participated in the Mock LISA Data Challenge 1. The challenges for which each group submitted results are marked by $(\bullet)$.

\begin{tabular}{|c|c|c|c|c|c|c|c|c|c|}
\hline \multirow[b]{3}{*}{ Group } & \multicolumn{7}{|c|}{ Galactic binaries } & \multirow{2}{*}{\multicolumn{2}{|c|}{$\begin{array}{c}\text { Massive } \\
\text { Black holes }\end{array}$}} \\
\hline & \multicolumn{3}{|c|}{ Single source } & \multicolumn{4}{|c|}{ Multiple sources } & & \\
\hline & $1.1 .1 \mathrm{a}$ & $1.1 .1 \mathrm{~b}$ & $1.1 .1 \mathrm{c}$ & 1.1.2 & 1.1 .3 & 1.1 .4 & 1.1 .5 & 1.2 .1 & 1.2 .2 \\
\hline AEI & $\bullet$ & $\bullet$ & $\bullet$ & $\bullet$ & $\bullet$ & $\bullet$ & $\bullet$ & & \\
\hline Ames & $\bullet$ & • & & $\bullet$ & $\bullet$ & & & & \\
\hline APC & $\bullet$ & & & & & & & & \\
\hline Goddard & & & & & & & & - & \\
\hline GLIG & & & $\bullet$ & & & & & & \\
\hline Królak & $\bullet$ & $\bullet$ & $\bullet$ & & $\bullet$ & & & & \\
\hline JPL/Caltech & & & & & & & & $\bullet$ & \\
\hline MT/AEI & & & & & & & & $\bullet$ & $\bullet$ \\
\hline MT/JPL & $\bullet$ & $\bullet$ & $\bullet$ & $\bullet$ & $\bullet$ & $\bullet$ & $\bullet$ & & \\
\hline UTB & $\bullet$ & $\bullet$ & $\bullet$ & $\bullet$ & $\bullet$ & $\bullet$ & & & \\
\hline
\end{tabular}

dwarf binaries (WDs), (1.2) supermassive black holes (SMBHs) and (1.3) extreme mass ratio inspirals (EMRIs). The problem of detection of EMRIs is considered more difficult than the others [6], so the deadline for submission of results for the 1.3 challenges is extended to June 2007. Consequently, in this paper we will discuss the results of Challenges 1.1 and 1.2.

The WD challenges consist of three single source data sets with the GW frequency around $1 \mathrm{mHz}$ (1.1.1a), $3 \mathrm{mHz}(1.1 .1 \mathrm{~b})$ and $10 \mathrm{mHz}$ (1.1.1c) and four multiple source data sets with isolated sources of known (1.1.2) and unknown (1.1.3) sky locations and frequencies, and overlapping sources with a low (1.1.4) and high (1.1.5) density of sources $(0.095$ and 0.35 , respectively) in frequency space. The SMBH challenges consist of two single source data sets. In one (1.2.1) the SMBH binary merges during the observation time, and in the other (1.2.2) the merger takes place between one and three months after the end of the data set. The source parameters were chosen with a balance between realism and computational efficiency as described in the announcement of the release [1].

\section{Overview of MLDC1 submissions}

Ten groups submitted results for MLDC1 by the deadline. These results have been posted on the MLDC website. They include the technical notes submitted by the challenge participants and the files with the 'best parameter fits' for the data sets. Table 1 provides a summary of the groups and their submissions for MLDC1.

With the exception of Challenges 1.1.5 and 1.2.2, every challenge data set was analysed by at least three groups. Here, we briefly summarize the approaches used by each group. More detailed descriptions from many of the groups can be found elsewhere in these proceedings [7-17] or in the technical notes on the MLDC web page [3]. Several groups used variations on matched filtering methods on many of the challenges. The Ames group at the NASA Ames Research Center employed a user-refined grid search on a number of the WD challenges. The AEI group and Andrzej Królak both used grid-based methods. The Global LISA Inference Group (GLIG), Montana-JPL and Montana-AEI groups employed variations on Markov Chain Monte Carlo (MCMC) methods [18]. The JPL-Caltech group used a multi-stage approach that combined time-frequency methods with grid-based and MCMC searches. The Montana-JPL group also used a genetic algorithm [19]. The APC group has also implemented a hierarchical 
approach which first matches the annual amplitude modulation and then follows with a fullmatched filtering. Two groups did not use matched filtering at all. The UTB group used a tomographic search that employed the Radon transform [20] while the Goddard group at the NASA Goddard Space Flight Center developed a time-frequency method that uses the Hilbert-Huang transform [21]. Although some of these groups have well-developed and mature algorithms, most groups are currently in various stages of development and so many of the entries are incomplete or suffer from known bugs which could not be hunted down before the December deadline. Some of the algorithms that are under development are meant to be part of a hierarchical search and so they only return a subset of the parameters needed to fully characterize the source.

\section{Assessment}

We evaluate the performance of all entries using the noise orthogonal pseudo $A$ and $E$ channels [22]:

$$
A=(2 X-Y-Z) / 3, \quad E=(Z-Y) / \sqrt{3},
$$

where $X, Y$ and $Z$ are the standard TDI variables. The wide variety of approaches and maturity of the algorithms makes it difficult to develop a single assessment that can adequately compare all entries. For those entries that have returned enough parameters to sufficiently generate a recovered waveform, we can compare the recovered waveform $h_{\text {rec }}$, with the waveform generated from the 'true' parameters $h_{\text {key }}$ using

$$
\Delta \chi^{2}=\frac{\left(h_{\mathrm{key}}-h_{\mathrm{rec}} \mid h_{\mathrm{key}}-h_{\mathrm{rec}}\right)}{D}
$$

where $(* \mid *)$ is the noise $\left(S_{n}\right)$ weighted inner product summed over channels $(i=A, E)$, defined by

$$
(a \mid b)=2 \int_{f_{\min }}^{f_{\max }} \frac{\sum_{i=A, E}\left(\tilde{a}_{i} b_{i}+a_{i} \tilde{b}_{i}\right)}{S_{n}} \mathrm{~d} f
$$

and $D$ is the dimension of the parameter space used to generate the templates. We realize that $\Delta \chi^{2}$ is not a perfect figure of merit as, for example, it does not account for deduced uncertainties in the recovered parameters. It is however easy to compute and is quite sufficient to indicate whether the recovered parameters differ greatly from those used for the key waveform. We can also compute the signal-to-noise ratio (SNR) for both $h_{\text {key }}$ and $h_{\text {rec }}$ using

$$
\mathrm{SNR}=\frac{(s \mid h)}{\sqrt{(h \mid h)}}
$$

and compare the recovered SNR with the key SNR. Finally, we calculate the correlation between $h_{\text {key }}$ and $h_{\text {rec }}$ with

$$
C=\frac{\left(h_{\mathrm{key}} \mid h_{\mathrm{rec}}\right)}{\sqrt{\left(h_{\mathrm{key}} \mid h_{\mathrm{key}}\right)\left(h_{\mathrm{rec}} \mid h_{\mathrm{rec}}\right)}} .
$$

The $\Delta \chi^{2}$ measure is sensitive to the difference in amplitude between $h_{\text {key }}$ and $h_{\text {rec }}$ while the SNR and correlation are both insensitive to the amplitude of $h_{\text {rec }}$.

Some groups reported a known ambiguity in the initial phase and polarization angles with results being given modulo $\pi / 2$ in the polarization angle and modulo $\pi$ in the initial phase [23]. The results would be indistinguishable under $\Delta \chi^{2}$, SNR, or $C$ tests, but would have been apparent in the parameter estimations errors. We have accounted for this ambiguity by choosing the values of $\psi$ and $\phi_{0}$ that minimize the error in $\psi$. If there are additional errors in 
Table 2. The performance of challenge entries on the single binary challenges as calculated using $\Delta \chi^{2}$, SNR and $C$. The correction of the initial phase by a factor of $\pi$ or $\pi / 2$ is indicated by an asterisk (*).

\begin{tabular}{|c|c|c|c|}
\hline Group & $\Delta \chi^{2}$ & SNR & $C$ \\
\hline \multicolumn{4}{|c|}{ Challenge 1.1.1a $\left(\mathrm{SNR}_{\mathrm{key}}=51.137\right)$} \\
\hline AEI & 8.095 & 50.604 & 0.989 \\
\hline Ames & 7.155 & 51.032 & 0.997 \\
\hline APC & 423.406 & -8.007 & -0.135 \\
\hline $\mathrm{APC}^{*}$ & 229.115 & 50.385 & 0.990 \\
\hline Królak & 778.888 & 0.933 & -0.004 \\
\hline Królak* & 1.036 & 51.038 & 0.999 \\
\hline MT/JPL (BAM) & 1.902 & 51.178 & 0.998 \\
\hline MT/JPL (GA) & 1.796 & 51.138 & 0.998 \\
\hline \multicolumn{4}{|c|}{ Challenge 1.1.1b $\left(\mathrm{SNR}_{\mathrm{key}}=37.251\right)$} \\
\hline AEI & 47.913 & 33.104 & 0.874 \\
\hline Ames & 64.371 & 32.067 & 0.822 \\
\hline Królak & 841.074 & -37.038 & -0.996 \\
\hline Królak* & 2.566 & 37.038 & 0.996 \\
\hline MT/JPL (BAM) & 7.735 & 36.856 & 0.980 \\
\hline MT/JPL (GA) & 8.371 & 36.808 & 0.979 \\
\hline \multicolumn{4}{|c|}{ Challenge $1.1 .1 \mathrm{c}\left(\mathrm{SNR}_{\mathrm{key}}=101.390\right)$} \\
\hline AEI & 2399.307 & -14.373 & -0.144 \\
\hline GLIG & 1788.991 & 14.496 & 0.142 \\
\hline Królak & 5997.595 & -98.126 & -0.968 \\
\hline Królak* & 97.603 & 98.126 & 0.968 \\
\hline MT/JPL (BAM) & 945.541 & 63.383 & 0.623 \\
\hline MT/JPL (GA) & 1376.143 & 43.564 & 0.424 \\
\hline
\end{tabular}

the returned value of $\phi_{0}$, they would remain after correcting for this ambiguity. These errors can arise from using different conventions for generating the waveform from the physical parameters or they can arise from determining the parameters in the form of a trigonometric function and then choosing the wrong quadrant when applying the inverse operation to obtain the angle. Obviously, a difference of $\pi$ in the initial phase can significantly degrade the performance of an entry as calculated using $\Delta \chi^{2}$, SNR or $C$. Consequently, we have also computed these measures with the initial phase shifted by $\pi$ if necessary.

Another measure of the success of a given algorithm is the accuracy with which it returns specific parameters. This approach allows us to also evaluate those entries which do not return enough parameters to generate $h_{\text {rec }}$. For each parameter $\lambda_{i}$, we can determine the difference between the key parameter and the recovered parameter using

$$
\Delta \lambda=\lambda_{\text {key }}-\lambda_{\text {rec }} \text {. }
$$

We note that it is not necessarily appropriate to use the Fisher information matrix (FIM) to determine the quality of parameter recovery. If the algorithm settles on a secondary maximum of the likelihood function or there are other systematic errors, then the results can be far from the regime of validity for the FIM approximation to expected errors in parameter estimation. For more details, see Vallisneri's review of the FIM [24].

The white dwarf binary challenges required the recovery of seven parameters to fully characterize each source. These parameters are the amplitude $\mathcal{A}$, the frequency $f$, the sky location $\theta, \phi$, the angle of inclination $\iota$, the polarization angle $\psi$ and the initial phase $\phi_{0}$. In table 2 , we list the values of the measures for each challenge entry for Challenges 1.1.1- 
Table 3. The performance of challenge entries on the single binary challenges as calculated using recovered parameter differences.

\begin{tabular}{|c|c|c|c|c|c|c|c|}
\hline Group & $\Delta f(\mathrm{nHz})$ & $\Delta \theta$ & $\Delta \phi$ & $\Delta \ln A$ & $\Delta \iota$ & $\Delta \psi$ & $\Delta \phi_{0}$ \\
\hline \multicolumn{8}{|c|}{ Challenge 1.1.1a } \\
\hline AEI & -1.208 & -0.018 & 0.001 & -0.078 & -0.101 & 0.157 & -0.065 \\
\hline Ames & -1.889 & -1.159 & 3.127 & 0.337 & 0.503 & 0.181 & -0.126 \\
\hline APC & 1.343 & -0.030 & -0.011 & 0.807 & 0.217 & 0.174 & 1.202 \\
\hline Królak & 0.980 & 0.028 & -0.008 & 0.113 & 0.180 & 0.208 & -2.089 \\
\hline MT/JPL (BAM) & -1.367 & -0.015 & -0.008 & -0.046 & -0.084 & 0.196 & -0.228 \\
\hline MT/JPL (GA) & -1.044 & -0.013 & -0.003 & -0.077 & -0.091 & 0.224 & -0.308 \\
\hline UTB & -3.209 & 0.143 & 0.603 & - & - & - & - \\
\hline \multicolumn{8}{|c|}{ Challenge $1.1 .1 \mathrm{~b}$} \\
\hline AEI & 0.399 & -0.049 & 0.001 & -0.009 & -0.045 & 0.020 & 0.432 \\
\hline Ames & -21.098 & -0.606 & 0.004 & 0.171 & 0.048 & 0.028 & 2.173 \\
\hline Królak & 0.341 & 0.037 & -0.004 & -0.112 & -0.042 & -0.042 & -3.098 \\
\hline MT/JPL (BAM) & 0.434 & -0.040 & 0.003 & -0.025 & -0.042 & 0.029 & 0.097 \\
\hline MT/JPL (GA) & 0.314 & -0.039 & 0.003 & -0.044 & -0.044 & 0.030 & 0.117 \\
\hline UTB & -4.299 & 0.198 & 0.007 & - & - & - & - \\
\hline \multicolumn{8}{|c|}{ Challenge $1.1 .1 \mathrm{c}$} \\
\hline AEI & -0.405 & 0.012 & -0.001 & 0.312 & -0.159 & 0.127 & 1.501 \\
\hline GLIG & 154.850 & 0.306 & 0.178 & 0.341 & 0.939 & 0.722 & 2.413 \\
\hline Królak & -5.210 & 0.059 & -0.010 & -0.194 & -0.268 & 0.451 & 2.747 \\
\hline MT/JPL (BAM) & -0.330 & 0.008 & -0.001 & 0.309 & 0.033 & -0.609 & 2.148 \\
\hline MT/JPL (GA) & 0.311 & 0.013 & -0.001 & 0.652 & -1.062 & -0.614 & 2.026 \\
\hline UTB & 8.577 & 0.139 & 0.066 & - & - & - & - \\
\hline
\end{tabular}

with the exception of the UTB entry. Since the UTB algorithm only returns frequency (in intervals of resolvable frequency bins $\sim 32 \mathrm{nHz}$ ) and sky position, it cannot be included in this comparison. However, it can be included in the comparison of parameter differences given in table 3. It should be noted that the GLIG cluster crashed before the completion of the algorithm and therefore the MCMC chain did not have the chance to burn into the final values. We also note that although the measures of Challenge 1.1.1c in table 2 seem to be quite bad, most of this is due to the fact that several groups used the low frequency approximation (which ignores the transfer function) in their analyses. Nonetheless, it is important to note that the accuracy in recovery of the sky positions and frequencies is still comparable to Challenges 1.1.1a and 1.1.1b. When corrected for an initial phase error, the entry by Królak (which incorporated the transfer function in the analysis) can be seen to have done quite well.

The multi-source challenges present a different problem for assessment, since there is the possibility of false positives and false negatives. Consider the possibility in which the recovered parameters for one binary out of many are wildly off. If one were to use the correlation between each recovered template and one of the source binaries, it is possible to count the recovered binary as a false positive and the true binary as a false negative. Given the phasing issues that were apparent in Challenges 1.1.1, it is quite likely that there will be several false positive/false negative pairs if such a correlation analysis is used. Consequently, we determine which recovered template goes with which source by looking for template/source pairs that are within one resolvable frequency bin of each other. The overall success of the recovery can be measured using the combined signal of the entire population of recovered binaries as $h_{\mathrm{rec}}$ and comparing with the entire population of the true source binaries as $h_{\text {key }}$. 
Table 4. The performance of challenge entries on the verification binary Challenge 1.1 .2 as calculated using $\Delta \chi^{2}$, SNR and $C$. Since every group returned the full 20 binaries, the dimension of the model is $D=140$. The SNR of the key is 544.952 .

\begin{tabular}{lcll}
\hline Group & $\Delta \chi^{2}$ & SNR & \multicolumn{1}{l}{$C$} \\
\hline AEI & 1443.59 & 339.262 & 0.624 \\
Ames & 227.007 & 516.471 & 0.948 \\
MT/JPL (BAM) & 19.02 & 544.165 & 0.998 \\
MT/JPL (GA) & 194.46 & 519.712 & 0.954 \\
\hline
\end{tabular}

Table 5. The performance of challenge entries on the isolated binary Challenge 1.1.3 as calculated using $\Delta \chi^{2}$, SNR and $C$. Since not all groups returned the full 20 binaries, the dimension of the model is calculated by $D=7 \times N_{\text {rec }}$, where $N_{\text {rec }}$ is the number of recovered binaries. The SNR of the key is 122.864 . The correction of the initial phase by a factor of $\pi$ or $\pi / 2$ is indicated by an asterisk $(*)$.

\begin{tabular}{lllll}
\hline Group & $\Delta \chi^{2}$ & SNR & $C$ & $N_{\text {rec }}$ \\
\hline AEI & 67.67 & 89.789 & 0.726 & 16 \\
Ames & 48.42 & 104.236 & 0.841 & 13 \\
Królak & 204.36 & 9.558 & 0.080 & 20 \\
Królak* & 150.95 & 38.770 & 0.323 & 20 \\
MT/JPL (BAM) & 44.81 & 98.838 & 0.797 & 19 \\
MT/JPL* (BAM) & 4.16 & 121.64 & 0.981 & 19 \\
MT/JPL (GA) & 19.66 & 113.797 & 0.914 & 18 \\
\hline
\end{tabular}

In this case, we note that the dimension $D$ of the recovered parameter space depends upon the number of recovered sources.

The Challenge 1.1.2 data set contained 20 'verification' binaries whose frequencies and sky location were given to the participants. Six of these binaries were taken from the list of known binaries available on Gijs Nelemans website [25] and the remaining 14 were simulated binaries. Of the four groups that submitted entries for Challenge 1.1.2, two of them did not use the additional information of sky location and frequency in their searches. The Montana/JPL group used the same search algorithms as they used in all of the 1.1 challenges, and the UTB group used their tomographic algorithm to obtain the frequency and sky location. The UTB group successfully recovered 17 of the 20 source binaries. The other three groups successfully recovered all 20 source binaries, although the AEI group had very low correlation with the two highest frequency binaries. This is understandable since the AEI group used the low frequency approximation (which is valid for frequencies below about $3 \mathrm{mHz}$ ) for calculating their templates and the two highest frequency binaries were at frequencies above $6 \mathrm{mHz}$. These two binaries are also responsible for most of the loss in correlation for this entry. The performance of the three groups that returned the complete parameterization of each binary recovered is given in table 4 .

Challenge 1.1.3 also contained 20 binaries isolated in frequency space between $0.5 \mathrm{mHz}$ and $10 \mathrm{mHz}$. In this challenge, all binaries were drawn from the simulation and all parameters were blind. Five groups submitted entries, although the UTB group again only provided frequency and sky location. The UTB group successfully identified 14 of the 20 binaries. The performance of the other four entries is given in table 5. The sky locations returned by each group are compared with the source positions in figure 1. 

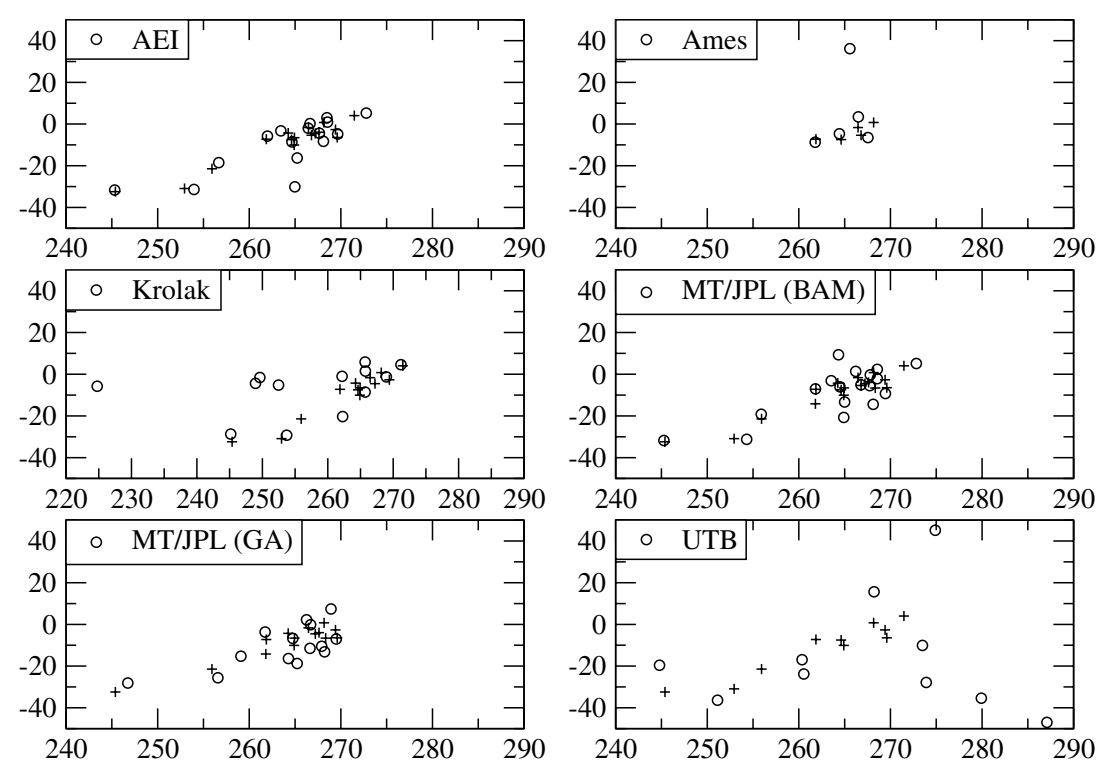

Figure 1. Recovered sky positions from each entry for Challenge 1.1.3. The source positions are indicated by + and the recovered positions are indicated by $\circ$. Each plot includes only those sources which are within a frequency bin of a recovered source. The plots are of ecliptic latitude versus longitude in degrees.

Table 6. The performance of challenge entries on the overlapping binary Challenges 1.1 .4 and 1.1.5 as calculated using $\Delta \chi^{2}$, SNR and $C$. Since not all groups returned the full number of binaries, the dimension of the model is calculated by $D=7 \times N_{\text {rec }}$, where $N_{\text {rec }}$ is the number of recovered binaries. $N_{\text {match }}$ is the number of recovered binaries that matched in frequency with a binary in the key.

\begin{tabular}{lcclll}
\hline Group & $\Delta \chi^{2}$ & SNR & $C$ & $N_{\text {rec }}$ & $N_{\text {match }}$ \\
\hline \multicolumn{5}{c}{ Challenge 1.1 .4} & $\left(\mathrm{SNR}_{\text {key }}=201.129\right)$ \\
AEI & 85.63 & 159.893 & 0.792 & 26 & 16 \\
MT/JPL (BAM) & 6.19 & 197.828 & 0.976 & 43 & 39 \\
\multicolumn{5}{c}{ Challenge 1.1 .5} & $\left(\mathrm{SNR}_{\text {key }}=178.261\right)$ \\
AEI & 519.21 & 116.822 & 0.654 & 5 & 4 \\
MT/JPL (BAM) & 11.96 & 172.582 & 0.963 & 27 & 23 \\
\hline
\end{tabular}

The remaining two white dwarf challenges each contained approximately 45 overlapping sources. Challenge 1.1 .4 contained 45 sources in a $15 \mu \mathrm{Hz}$ band starting at $3 \mathrm{mHz}$, while Challenge 1.1 .5 contained 33 sources in a $\pm 1.5 \mu \mathrm{Hz}$ band centred on $3 \mathrm{mHz}$. Challenge 1.1.4 had an average density of 0.095 sources per resolvable frequency bin, and Challenge 1.1.5 had an average density of 0.35 sources per bin. These challenges were actually more difficult than these source densities might indicate since in both cases there were at least three frequency bins which contained at least two binaries each. There was one case in Challenge 1.1.5 with three binaries in one frequency bin. Despite this additional complication, both groups managed to recover a respectable number of sources that matched with binaries in the key. The performance of the two groups that submitted complete parameter sets for each binary recovered is listed in table 6 . 
Table 7. The performance of challenge entries on the supermassive black hole binary Challenges 1.2.1 and 1.2.2. The results after a correction of the initial phase are indicated by an asterisk (*).

\begin{tabular}{|c|c|c|c|c|c|}
\hline Group & $\Delta \chi^{2}$ & SNR & $O_{A}$ & $O_{E}$ & $\max _{\phi_{0}}\left(O_{X}\right)$ \\
\hline \multicolumn{6}{|c|}{ Challenge 1.2.1 $\left(\mathrm{SNR}_{\mathrm{key}}=667.734\right)$} \\
\hline JPL & 261.48 & 664.47 & 0.994 & 0.996 & 0.9955 \\
\hline MT/AEI & 10289.29 & 524.29 & 0.790 & 0.791 & 0.9998 \\
\hline $\mathrm{MT} / \mathrm{AEI}^{*}$ & 105.50 & 662.87 & 0.998 & 0.998 & 0.9998 \\
\hline \multicolumn{6}{|c|}{ Challenge 1.2.2 $\left(\mathrm{SNR}_{\mathrm{key}}=104.19\right)$} \\
\hline MT/AEI & 1.41 & 104.29 & 0.997 & 0.998 & 0.9955 \\
\hline
\end{tabular}

The supermassive black hole challenges required recovery of nine parameters describing the source: the chirp mass $\mathcal{M}$, the reduced mass $\mu$, the luminosity distance $D_{L}$, the time of coalescence $t_{c}$, the sky location $\theta, \phi$, the initial angle of inclination $\iota$, the initial polarization angle $\psi$ and the initial orbital phase $\phi_{0}$. As with the white dwarf challenges, the quality of the recovered signal can be described by the measures given in equations (2) and (4). In place of the correlation, we compute several overlaps using

$$
O_{\alpha}=\frac{\left(h_{\mathrm{key}}^{\alpha} \mid h_{\mathrm{rec}}^{\alpha}\right)}{\sqrt{\left(h_{\mathrm{key}}^{\alpha} \mid h_{\mathrm{key}}^{\alpha}\right)\left(h_{\mathrm{rec}}^{\alpha} \mid h_{\mathrm{rec}}^{\alpha}\right)}}
$$

where $\alpha$ denotes the particular TDI channel being used. In order to mitigate the effects of a possible error in the initial phase, we have also computed $O_{X}$, maximized over the phase:

$$
\max _{\phi_{0}}\left(O_{X}\right)=\sqrt{\left(h_{\text {rec }}^{X} \mid h_{\text {key }}^{X}\left(\phi_{0}=0\right)\right)^{2}+\left(h_{\text {rec }}^{X} \mid h_{\text {key }}^{X}\left(\phi_{0}=\pi / 2\right)\right)^{2}} .
$$

There were two groups that returned a full characterization of the signal for Challenge 1.2.1. The Montana/AEI group had a constant phase difference, and when this phase is corrected the performance of both the JPL/Caltech and Montana/AEI groups is quite good. The Goddard group is developing a new algorithm using the Hilbert-Huang transform that is in a very preliminary stage and has only returned $\mathcal{M}$ and $t_{c}$ for this challenge. Because of a known secondary maximum in sky location, we also check the antipodal sky position:

$$
\theta \rightarrow-\theta, \quad \phi \rightarrow \phi \pm \pi \text {. }
$$

However, this adjustment also requires a change in the values of the inclination and polarization angles as well. This is accomplished by substituting the initial returned values of $\theta, \phi, \iota$ and $\psi$ into

$$
\begin{aligned}
& \cos \iota=\cos \theta \sin \Theta \cos (\phi-\Phi)+\cos \Theta \sin \theta \\
& \tan \psi=\frac{\sin \theta \cos (\phi-\Phi) \sin \Theta-\cos \Theta \cos \theta}{\sin \Theta \sin (\phi-\Phi)},
\end{aligned}
$$

and solve these equations for the orientation angles of the orbital angular momentum vector, $(\Theta, \Phi)$. Once we have the values of $(\Theta, \Phi)$, we then use these values and the antipodal sky position from equations (9) in the above equations (10) and (11) to determine the new values of $\iota$ and $\psi$. Once these have been found, the new values can be used to provide a more realistic estimate of the error in the returned values. We have applied this transformation to the JPL/Caltech entry and also adjusted the polarization phase for the Montana/AEI entry in Challenge 1.2.1. Only one group (Montana/AEI) submitted an entry for Challenge 1.2.2. The measures for each submission under both of these challenges are given in table 7 . We have also determined the errors in the recovered parameters for all entries in Challenges 1.2.1 and 1.2.2. These are presented in table 8 . 
Table 8. The performance of challenge entries on the supermassive black hole binary Challenges 1.2.1 and 1.2.2 on the estimation of recovered parameters. The angles are absolute errors, all other errors are relative.

\begin{tabular}{|c|c|c|c|c|c|c|c|c|c|}
\hline Group & $\begin{array}{l}\Delta \mathcal{M} / \mathcal{M} \\
\left(\times 10^{-4}\right)\end{array}$ & $\Delta \mu / \mu$ & $\Delta D_{L} / D_{L}$ & $\begin{array}{l}\Delta t_{c} / t_{c} \\
\left(\times 10^{-6}\right)\end{array}$ & $\Delta \theta$ & $\Delta \phi$ & $\Delta \iota$ & $\Delta \psi$ & $\Delta \phi_{0}$ \\
\hline \multicolumn{10}{|c|}{ Challenge 1.2.1 (reported values) } \\
\hline JPL & 7.35 & 0.011 & 1.101 & 3.35 & 1.030 & -3.170 & 1.32 & -2.65 & 0.004 \\
\hline $\mathrm{MT} / \mathrm{AEI}$ & 0.98 & 0.001 & 0.042 & 0.26 & 0.001 & 0.001 & 0.02 & 3.14 & 0.004 \\
\hline Goddard & 434.00 & - & - & 113.00 & - & - & - & - & - \\
\hline \multicolumn{10}{|c|}{ Challenge 1.2.1 (angle adjusted values) } \\
\hline JPL & 7.35 & 0.011 & 1.101 & 3.35 & -0.043 & -0.032 & -0.58 & -0.31 & 0.004 \\
\hline $\mathrm{MT} / \mathrm{AEI}$ & 0.98 & 0.001 & 0.042 & 0.26 & 0.001 & 0.001 & 0.02 & -0.00 & 0.004 \\
\hline \multicolumn{10}{|c|}{ Challenge 1.2.2 } \\
\hline $\mathrm{MT} / \mathrm{AEI}$ & 3.09 & 0.037 & 0.273 & 182.00 & 0.019 & 0.005 & -0.71 & -2.16 & -0.002 \\
\hline
\end{tabular}

\section{Conclusions}

The first round of the Mock LISA Data Challenges successfully attracted over ten groups to work on the problem of LISA data analysis. These groups attacked several of the challenges with a variety of different approaches. The algorithms and codes used in the challenges were at different levels of maturity and completeness of the pipelines. Nonetheless, all challenges had at least one entry which successfully characterized the signal to better than $95 \%$ when assessed via the correlation with phasing ambiguities accounted for. In the overlapping source challenge 1.1.5, one group was able to recover true binaries at a source density of $\sim 0.25$. Most groups also discovered small bugs or discrepancies in definitions of some of the parameters used to characterize the signal. The first round entries were a success, especially considering that most groups had less than five months to work out interfacing issues and assess their codes. Those groups that participated in round 1 have begun implementing the lessons learned for use in round 2 [26], which is another successful outcome of the challenges. The MLDC Task Force has also begun to address the issues that have been raised as regards assessment of the entries. As the assessments become more refined in future challenges, we anticipate developing assessments for the true LISA data for which there is no key file.

\section{Acknowledgments}

MB acknowledges funding from NASA Grant NNG04GD52G. MB, SM and RN were supported by the NASA Center for Gravitational Wave Astronomy at University of Texas at Brownsville (NAG5-13396). N Christensen acknowledges funding from NSF Grant PHY0553422 and the Fulbright Scholar Program. N Cornish, J Crowder and EP acknowledge funding from NASA Grant NNG05GI69G. J Crowder, CC and MV carried out this work at JPL, Caltech, under contract to NASA. MV is grateful for support from the Human Resources Development Fund program at JPL. IM would like to thank the Brinson Foundation, NASA Grant NNG04GK98G and NSF Grant PHY-0601459. DB was supported by NSF Grant PHY-0601459 and the LIGO Laboratory. LIGO was constructed by the California Institute of Technology and Massachusetts Institute of Technology with funding from the National Science Foundation and operates under cooperative agreement PHY-0107417. The work of AK was supported in part by grant 1 P03B 02927 from the Polish Ministry of Science and 
Information Technology. AK would like to acknowledge hospitality of Max Planck Institute for Gravitational Physics in Potsdam, Germany, where part of his work was done. AV was partially supported by the Packard Foundation and the NSF.

\section{References}

[1] Arnaud K A et al 2006 AIP Conf. Proc. 873619 (Preprint gr-qc/0609105)

[2] Arnaud K A et al 2006 AIP Conf. Proc. 873625 (Preprint gr-qc/0609106)

[3] Mock LISA Data Challenge Homepage astrogravs.nasa.gov/docs/mldc

[4] Mock LISA Data Challenge Task Force wiki www.tapir.caltech.edu/dokuwiki/listwg1b:home

[5] Mock LISA Data Challenge Task Force, 'Document for Challenge 1', svn.sourceforge.net/viewvc/lisatools/ Docs/challenge1.pdf

[6] Gair J R, Barack L, Creighton T, Cutler C, Larson S L, Phinney E S and Vallisneri M 2004 Class. Quantum Grav. 211595

[7] Auger G et al 2007 Class. Quantum Grav. submitted

[8] Camp J B, Cannizzo J K and Numata K 2007 Phys. Rev. D 75061101 (Preprint gr-qc/0701148)

[9] Crowder J and Cornish N 2007 Class. Quantum Grav. 24 S575

[10] Brown D et al 2007 Class. Quantum Grav. 24 S595

[11] Królak A 2007 Class. Quantum Grav. submitted

[12] Nayak R K, Mohanty S D and Hayama K 2007 Class. Quantum Grav. 24 S587

[13] Porter E and Cornish N 2007 Class. Quantum Grav. 24 S501

[14] Prix R and Whelan J T 2007 Class. Quantum Grav. 24 S565

[15] Roever C et al 2007 Class. Quantum Grav. 24 S521

[16] Stroeer A et al 2007 Class. Quantum Grav. 24 S541

[17] Thompson D E and Thirumalainambi R 2007 Class. Quantum Grav. submitted

[18] Gilks W R, Richardson S and Spiegelhalter D J 1996 Markov Chain Monte Carlo in Practice (Boca Raton, FL: Chapman and Hall/CRC Press)

[19] Holland J 1975 Adaptation in Natural and Artificial Systems (Ann Arbor, MI: University of Michigan Press)

[20] Deans S R 1983 The Radon Transform and Some of its Applications (New York: Wiley)

[21] Huang N et al 1998 Proc. R. Soc. Lond. 454903

[22] Prince T A, Tinto M, Larson S L and Armstrong J W 2002 Phys. Rev. D 66122002

[23] Cornish N J and Larson S L 2003 Phys. Rev. D 67103001

[24] Vallisneri M 2007 Use and abuse of the Fisher information matrix in the assessment of gravitational-wave parameter-estimation prospects Preprint gr-qc/0703086

[25] Nelemans G LISA Wiki www.astro.kun.nl/ nelemans/dokuwiki

[26] Arnaud K A et al 2007 Class. Quantum Grav. 24 S551 